\title{
HilA-like regulators in Escherichia coli pathotypes: the YgeH protein from the enteroaggregative strain 042
}

\author{
Mário Hüttener ${ }^{1}$, Manuela Dietrich ${ }^{1}$, Sònia Paytubi ${ }^{2}$ and Antonio Juárez ${ }^{1,2^{*}}$
}

\begin{abstract}
Background: The HilA protein is the master regulator of the Salmonella pathogenicity island 1 (SPI1). EilA and YgeH proteins show a moderate similarity to HilA and are encoded in pathogenicity islands from several E. coli strains, both pathogenic and non-pathogenic. In the present work we characterize the $\mathrm{YgeH}$ protein from the enteroaggregative E. coli strain 042 (locus tag EC042_3050).

Results: We show that both E. coli $042 \mathrm{YgeH}$ and EilA proteins are able to functionally replace HilA in Salmonella. Interestingly, this is not the rule for all YgeH proteins: the YgeH protein from the enterohaemorragic E. coli strain O157 appears to be non-functional. ygeH expression is not influenced by growth osmolarity or temperature, and moderately increases in cells entering the stationary phase. H-NS represses ygeH expression under all growth conditions tested, and binds with specificity to the ygeH promoter region. As expected, expression of ETT2 (Escherichia coli type 3 secretion system 2) genes requires YgeH: ETT2 operons are downregulated in a ygeH mutant. Accordingly, since H-NS represses ygeH expression, ETT2 expression is significantly increased in an hns mutant.
\end{abstract}

Conclusion: E. coli $042 \mathrm{YgeH}$ protein is functional and able to replace HilA in Salmonella. ETT2 gene expression requires $\mathrm{YgeH}$ activity which, in turn, is subjected to H-NS silencing.

Keywords: HilA, YgeH, E. coli 042, H-NS

\section{Background}

Diarrhoeal disease is the second leading cause of infant mortality under the age of 5 worldwide [1]. Enteropathogenic Escherichia coli (EPEC) is a human pathogen of the small intestine and is a significant cause of infantile diarrhea [2]. EPEC were recognized as pathogens several decades ago [3]. Later on, EPEC and enteroaggregative $E$. coli (EAEC) were distinguished from each other on the basis of their patterns of adherence to HEp-2 cells. Unlike the localised "microcolonyforming" pattern of adherence associated with EPEC, EAEC display a characteristic aggregative or "stacked-brick" pattern of adherence [4]. Nataro et al. demonstrated a significant association of EAEC with diarrhea in a case control study of children in Chile [4]. Immediately following the discovery of EAEC as a category of pathogenic $E$.

\footnotetext{
*Correspondence: ajuarez@ub.edu

${ }^{1}$ Institute for Bioengineering of Catalonia (IBEC), Barcelona, Spain

${ }^{2}$ Department of Microbiology, Faculty of Biology, University of Barcelona, Barcelona, Spain
}

coli, several epidemiological reports casted doubt on the pathogenic nature of EAEC [5]. Nevertheless, a volunteer study provided evidence for EAEC strain 042 eliciting diarrhea in the majority of volunteers [6]. Since then many studies have supported the association of EAEC and diarrhea in both developing countries and industrialized nations. Thus, EAEC have been significantly associated with (i) endemic diarrhea in infants in developing and industrialised nations, (ii) persistent diarrhea in HIV-positive patients, (iii) traveller's diarrhea, (iv) food/ water-borne outbreaks, and (v) sporadic cases of diarrhea [6]. Furthermore, the increasing number of reports in which EAEC is implicated as the agent mediating diarrhea suggests that $E$. coli strains belonging to this pathotype are important emerging pathogens [7]. A large number of virulence factors has been associated with clinical illness in epidemiologic studies [8]. It is also remarkable that EAEC strains are heterogeneous [9]. As not all strains of EAEC tested elicited diarrhea, the EAEC strain 042 which caused diarrhea in the volunteer 
study became the prototypical EAEC strain for the study of virulence factors and EAEC pathogenicity [6]. Its genome has been sequenced [10] and the virulence factors characterized.

Success for a bacterial pathogen to cause disease requires not only the display of several virulence determinants, but also a precise control of their expression, such that each is expressed at the appropriate time and place in the host. A good example for that is expression of the Salmonella pathogenicity island 1 (SPI1). Several environmental factors and regulators have been identified as affecting SPI1 [11]. It has been shown that SPI1 environmental regulation converges in the modulation of the hilA gene [12]. hilA is located on SPI1 and encodes the HilA protein, a member of the OmpR/ToxR family of regulators [13,14]. HilA regulation itself is very complex and responds to several stimuli [15-17]. Both positive and negative regulators dictate appropriate HilA levels, which, in turn, result in activation or repression of the SPI-encoded effectors.

A genomic analysis of the type 3 secretion system (TTSS) from E. coli showed that two different genomic islands encoded hilA homologues [18]. The eip island, identified in the E. coli 042 genome encodes, in addition to different effectors, the eilA gene, a HilA-like regulator [19]. In addition, the ETT2 pathogenicity island encodes the $y g e H$ gene, which shows significant similarity to HilA. This latter pathogenicity island also encodes a TTSS and different effectors. Remarkably, whereas ETT2 appears to be functional in E. coli 042, it has been subjected in the rest of the strains analysed to varying degrees of mutational attrition that results in a non-functional TTSS.

The role of EilA, the HilA-homologue encoded in the genome of the enteroaggregative strain 042 was characterized a few years ago [19]. EilA expression was reported to modulate expression of different genes of the eip island (i.e., eipB, eipC, eipD, eicA and eaeX), as well as of two genes of a pathogenicity island not linked to eip (ETT2). EilA defect was associated to alterations in adherence to epithelial cells and to biofilm formation. A role for EilA modulating expression of the TTSS and effectors of different chromosomal islands in EAEC was suggested. In contrast to EilA, the regulatory role of YgeH has not been hitherto characterized. We study in this report YgeH. We show that it is able to compensate for HilA depletion in Salmonella. We also study its regulation, and describe some of its target genes in the ETT2 pathogenicity island from strain 042.

\section{Methods}

\section{Bacterial strains, plasmids and culture conditions}

All bacterial strains and plasmids used in this study are listed in Table 1. Cultures were normally grown in Luria-Bertani (LB) medium (10 g NaCl, $10 \mathrm{~g}$ tryptone
Table 1 Bacterial strains and plasmids used in this study

\begin{tabular}{|c|c|c|}
\hline Strain or plasmid & Description & $\begin{array}{l}\text { Source or } \\
\text { reference(s) }\end{array}$ \\
\hline \multicolumn{3}{|l|}{$\begin{array}{l}\text { S. enterica serovar } \\
\text { Typhimurium }\end{array}$} \\
\hline SV5015 & SL1344 hist & J. Casadesús \\
\hline $\mathrm{MH}-\mathrm{C} 1$ & SV5015 hilA::Cm & {$[20]$} \\
\hline MHS-1 & SV5015 $\triangle$ hilA & {$[20]$} \\
\hline SV5258 & SV5015 ФinvF-lacZY & J. Casadesús \\
\hline SV5258HilA & SV5258 $\triangle$ hilA::Cm & This work \\
\hline MHS-2 & SV5015 sipA::3XFLAG & This work \\
\hline MHS-3 & MHS-1 sipA::3XFLAG & This work \\
\hline LB5000 & $\mathrm{rLT}^{-} \mathrm{rSA}^{-} \mathrm{rSB} \mathrm{B}^{-}$ & {$[21]$} \\
\hline \multicolumn{3}{|l|}{ Escherichia coli } \\
\hline E. coli O157:H7 & $\begin{array}{l}\text { does not produce either Shiga-like } \\
\text { toxin I or II (ATCC } N^{\circ} 43888 \text { ) }\end{array}$ & ATCC \\
\hline AAG1 & MG1655 $\triangle$ LacZYA & {$[22]$} \\
\hline AAG1H & AAG1 $\Delta h n s$ & This work \\
\hline AAG1IHFA & AAG1 $\triangle i h f A:: C m$ & This work \\
\hline AAG1IHFB & AAG1 $\triangle i h f B:: \mathrm{Cm}^{-}$ & This work \\
\hline AAG1HA & AAG1 $\triangle h n s \triangle i h f A:: C m$ & This work \\
\hline $\mathrm{AAG} 1 \mathrm{HB}$ & AAG1 $\triangle h n s \triangle i h f B:: C m$ & This work \\
\hline AAG1入RSygeH & AAG1 $\lambda$ RSygeH integrated attB $\mathrm{Km}^{r}$ & This work \\
\hline AAG1H入RSygeH & $\mathrm{AAG} 1 \mathrm{H}+\lambda \mathrm{RSygeH}$ & This work \\
\hline AAG1HAגRSygeH & $\mathrm{AAG} 1 \mathrm{HA}+\lambda \mathrm{RSygeH}$ & This work \\
\hline AAG1HBגRSygeH & $\mathrm{AAG} 1 \mathrm{HB}+\lambda \mathrm{RSygeH}$ & This work \\
\hline EAEC 042 & $\mathrm{Cm}^{r} \mathrm{Sm}^{r} \mathrm{~T} c^{r}$ & I. Henderson \\
\hline 042HNS & EAEC $042 \Delta h n s$ & This work \\
\hline 042YgeH & EAEC $042 \Delta y g e H$ & This work \\
\hline 042YgeH3X & EAEC 042 ygeH::3xFLAG & This work \\
\hline 042YgeH3XHNS & 042YgeH3X $\Delta h n s$ & This work \\
\hline
\end{tabular}

Plasmids

pKD4

pKD3

pKD46

pCP20

pSUB11

pBAD18

PBADHilA

pBADEilA

pBADYgeH042

pBADYgeHO157

pRS551

pYgeH042

\begin{tabular}{|c|c|}
\hline bla FRT ahp FRT PS1 PS2 oriR6K $\mathrm{Km}^{\mathrm{r}} \mathrm{Cb}^{\mathrm{r}}$ & {$[23]$} \\
\hline bla FRT cat FRT PS1 PS2 oriR6K $\mathrm{Cm}^{r} \mathrm{Cb}^{r}$ & {$[23]$} \\
\hline bla $\mathrm{P}_{\mathrm{BAD}}$ gam bet exo pSC101 oriTs Cbr & {$[23]$} \\
\hline bla cat cl857 $\mathbb{I P}_{\mathrm{R}} f l p$ pSC101 oriTs Cb ${ }^{r}$ & {$[24]$} \\
\hline $\begin{array}{l}\text { 3xFLAG- and } \mathrm{Km}^{\mathrm{r}} \text {-coding template } \\
\text { vector }\end{array}$ & {$[25]$} \\
\hline $\operatorname{rep}_{\mathrm{pMB} 1} \mathrm{p}_{\text {araBAD }} \mathrm{Cb}^{\mathrm{r}}$ & {$[26]$} \\
\hline pBAD18 + hilA $A_{S V 5015}$ & This work \\
\hline pBAD18 + eilA $A_{\text {EAEC042 }}$ & This work \\
\hline $\mathrm{pBAD} 18+y g e H_{\text {EAEC042 }}$ & This work \\
\hline $\mathrm{pBAD} 18+y g e H_{\mathrm{O} 157: \mathrm{H} 7}$ & This work \\
\hline Promoterless vector lac $Z \mathrm{Km}^{\mathrm{r}} \mathrm{Cb}^{\mathrm{r}}$ & {$[27]$} \\
\hline $\begin{array}{l}\text { ygeH } 042 \text { promoter cloned into } \\
\text { pRS551 }\end{array}$ & This work \\
\hline
\end{tabular}

bla FRT ahp FRT PS1 PS2 oriR6K $\mathrm{Km}^{r} \mathrm{Cb}^{r}$

bla $\mathrm{P}_{\mathrm{BAD}}$ gam bet exo pSC101 oriTs $\mathrm{Cb}^{r}$

3xFLAG-and Km'-coding template

rep pMB1 $_{1} \mathrm{p}_{\text {araBAD }} \mathrm{Cb}$

This work

This work

$[27]$

yge $_{042}$
pRS551 
and $5 \mathrm{~g}$ yeast extract per litre) at the indicated temperature with vigorous shaking at 200 r.p.m. (Innova 3100, New Brunswick Scientific). Where indicated, LB medium without $\mathrm{NaCl}$ (0 g per liter), or D-MEM supplemented 1:1 with LB together with added $0.2 \%$ glucose (eilA induction conditions described by [19]) was used. Antibiotics were used at the following concentrations: kanamycin $\left(50 \mu \mathrm{g} \mathrm{ml}^{-1}\right)$, carbenicillin $\left(100 \mu \mathrm{g} \mathrm{ml}^{-1}\right)$ and chloramphenicol $\left(25 \mu \mathrm{g} \mathrm{ml}^{-1}\right)$.

To construct plasmids pBADEilA, pBADYgeH ${ }_{042}$, pBADHilA and pBADYgeH ${ }_{\mathrm{O} 157}$, eilA and $y g e H$ genes of strain E. coli 042, hilA gene of $S$. Typhimurium SL1344 strain and $y g e H$ gene from $E$. coli $\mathrm{O} 157 \mathrm{H} 7$ were amplified using oligonucleotides EILAKPNI5-EILAHINDIII3, YGE HKPNI5-YGEHHINDIII3, HILABADFW-HILABADRV and YGEHO157XbaFW-YGEHO157HindRV, respectively (see Additional file 1). The oligonucleotides add KpnI and HindIII sites, to the eilA and ygeH genes from E. coli 042, EcoRI and XbaI sites to the hilA gene and XbaI HindIII sites to $y g e H$ gene from E. coli O157:H7. The corresponding KpnI-HindIII, EcoRI-XbaI and XbaI-HindIII PCR fragments were cloned into pBAD18 digested with the same enzymes, resulting in plasmids pBADEilA, $\mathrm{pBADYgeH}_{042}$, $\mathrm{pBADHilA}$ and $\mathrm{pBADYge} \mathrm{H}_{\mathrm{O} 157}$, respectively.

\section{Genetic manipulations}

Standard molecular and genetic procedures were performed as described by [28]. Enzymes were used according to the manufacturer's recommendations. Introduction of plasmids into Escherichia coli and $S$. Typhimurium strains was performed by electroporation of 10\% glycerol-washed cells using an Eppendorf gene pulser (Electroporator 2510). Plasmids isolated from E. coli were first passaged through the restriction-deficient $S$. Typhimurium strain LB5000 [21] before transformation of $S$. Typhimurium SL1344 competent cells.

Chromosomal deletions of hilA from S. Typhimurium, hns, ihfA and ihfB from E. coli AAG1 and ygeH and hns from $E$. coli 042 were obtained by the $\lambda$ Red recombinant method as previously described [23]. The antibioticresistance determinant of plasmid pKD4 was amplified using oligonucleotides HNSP1/HNSP2, YGEHP1/YGEHP2 for hns and ygeH genes, and, in the case of hilA, ihfA and ihfB deletions, the chloramphenicol resistance was amplified using as template the pKD3 plasmid and the oligonucleotides HIIAP1/HILAP2 and IHFAP1/IHFAP2, IHFBP1/ IHFBP2 (see Additional file 1). Mutants were selected on LB plates containing the appropriate selection marker, and the successful deletion of the gene was confirmed by PCR using the primers CAT-C1 and CAT-C2 (chloramphenicol resistance; $\mathrm{Cm}^{\mathrm{r}}$ ) or $\mathrm{KT}$ and $\mathrm{K} 2$ (kanamycin resistance; $\mathrm{Km}^{\mathrm{r}}$ ) in combination with specific primers located in the remaining gene sequence nearby (Additional file 1, P1UP/P2DOWN series oligonucleotides). When necessary, the antibiotic resistance was then eliminated by transforming the mutant strain with plasmid pCP20 and subsequent incubation at $43^{\circ} \mathrm{C}$ for two passages according to [23]. The double deletions were obtained by combining one previously deletion with other deletion associated with an antibiotic resistance.

Chromosomal insertions of 3XFLAG sequences to the sipA and ygeH genes were obtained by a modification of the $\lambda$ Red recombinant method, as described by [25]. The antibiotic-resistance determinant of plasmid pSUB11 was amplified using oligonucleotides SIPA3XP1/SIPA3XP2 and YGEH3XP1/YGEH3XP2 for sipA and $y g e H$, respectively (Additional file 1). Mutants were selected on LB plates containing kanamycin, and successful 3XFLAG insertion was confirmed by PCR using the oligonucleotides KT and K2 (kanamycin resistance; $\mathrm{Km}^{\mathrm{r}}$ ) in combination with specific oligonucleotides located in the remaining gene sequence nearby (Additional file 1, 3XP1UP/3XP2DOWN series oligonucleotides). The SV5015SipA strain was used as a donor strain to transfer the SipA::3xFLAG fusion to strain SV5015HilA using phage P22 HT [29], generating strain SV5015SH. The chromosomal fusion YgeH::3xFLAG was constructed in the parental strain E. coli 042 and the isogenic mutant $\Delta h n s$, generating 042YgeH3X and 042YgeH3XHNS strains, respectively.

\section{Construction of an ygeH::IacZ transcriptional fusion}

A transcriptional ygeH:: lac Z fusion was constructed by cloning the promoter region of the $y g e H$ gene from EAEC 042 into the pRS551 plasmid. A single copy of the fusion was then inserted into the chromosome of the AAG1 strain by using the bacteriophage $\lambda R S 45$ as described by [27]. First of all, the promoter region of the $y g e H$ gene was amplified using the oligonucleotides YGEHBAMHI5/YGEHBAMHI3 (Additional file 1) that add a BamHI site, and the resulting PCR product was purified using the kit High Pure PCR Product Purification Kit (Roche). Subsequently, the purified PCR product was digested with BamHI enzyme (Fermentas) and ligated into the BamHI-digested pRS551, generating the plasmid $\mathrm{pYgeH}$. The transcriptional fusion ygeH::lacZ cloned into the plasmid pYgeH was then transferred to the $\lambda R S 45$ bacteriophage according to [27] and integrated as a single copy into the attB locus of the AAG1 chromosome. To confirm the correct insertion from the selected resulting colonies, a PCR screening was performed according to [30].

\section{Beta-galactosidase assay}

$\beta$-galactosidase activity measurements were performed as described by [31].

\section{Cell-free supernatant preparation}

The supernatants from cultures were obtained utilizing trichloroacetic acid (TCA) as agent for protein precipitation 
and acetone for removal traces from TCA treatment. The cultures were grown in LB medium until O.D.600nm at 2.0, and then $10 \mathrm{ml}$ were centrifuged at $6.000 \mathrm{rpm}$ for 10 minutes at room temperature. The supernatant was filtered with a $0.22 \mu \mathrm{m}$ pore size filter (Millex GP, Millipore) and proteins were precipitated by adding TCA at a final concentration of $10 \%$ and maintaining samples on ice for 1 hour. The samples were then centrifuged for 30 minutes at $13.400 \mathrm{rpm}$ at $4^{\circ} \mathrm{C}$, the supernatants were discarded and $0.5 \mathrm{ml}$ of acetone was added. The samples were centrifuged once more as described above. After removal of acetone the pellet was air-dried for 10 minutes and the samples were reconstituted in sample buffer (Laemmli sample buffer, Bio-Rad).

\section{SDS-PAGE and western blotting}

Protein samples were analysed by SDS-PAGE at $10 \%$ and $12.5 \%$. Proteins were transferred from the gels to PVDF membranes. Western blot analysis was performed with monoclonal antibodies raised against FLAG-epitope (1:10.000, Sigma) or against GAPDH (1:2000, Thermo Scientific) and horseradish peroxidase-conjugated goat anti-mouse IgG (1:2500, Promega). Detection was performed by enhanced chemiluminescence using Quantity One software (Bio-Rad).

\section{RNA isolation}

Total RNA was extracted from bacteria using the RNeasy Mini kit (Qiagen) according to the manufacturer's instruction. Potential traces of DNA were removed by digestion with DNase I (Turbo DNA-free, Ambion), according to the manufacturer's protocol. RNA concentration and RNA quality were measured using a Nano- Drop 1000 (Thermo Fisher Scientific).

\section{Real-time qRT-PCR}

Real-time quantitative reverse transcription-PCR (qRT-PCR) was performed as previously described [32]. Briefly, 1 $\mu \mathrm{g}$ of total RNA was reverse transcribed to generate cDNA using the High-capacity cDNA Reverse Transcription kit (Applied Biosystems) as recommended by the manufacturer. As a control, parallel samples were run in which reverse transcriptase was omitted from the reaction mixture. Oligonucleotides complementary to the genes of interest were designed using primer3 software. Real-time PCR using SYBR green PCR master mix (Applied Biosystems) was carried out on the ABI Prism 7700 sequence detection system (Applied Biosystems). After analysis of amplification plots with the ABI Prism sds software package, relative quantification of gene transcription was performed using the comparative threshold cycle (CT) method. The relative amount of target cDNA was normalized using the gapA gene as an internal reference standard.

\section{Electrophoretic mobility shift assay (EMSA)}

For EMSA, a 228 bp fragment corresponding to the promoter region of the yge $\mathrm{H}$ operon (nucleotides -207 to +21 ) was amplified using oligonucleotides YGEHBAMHI5/ YGEHBAMHI3 (Additional file 1). For each reaction, 50 ng DNA were mixed with increasing concentrations of the purified H-NS-His protein in binding buffer $(250 \mathrm{mM}$ HEPES, pH 7.4, $350 \mathrm{mM} \mathrm{KCl,} 5$ mM EDTA, 5 mM DTT, $500 \mu \mathrm{g} \mathrm{BSA} \mathrm{ml}{ }^{-1}, 25 \%$ glycerol). After incubation for $30 \mathrm{~min}$ at room temperature, $20 \mu \mathrm{l}$ of samples were separated on $5 \%$ polyacrylamide/0.5× TBE gel. The bands were stained using ethidium bromide and visualized using Quantity One software (Bio-Rad). For competitive EMSA, the fragment corresponding to the $y g e H$ promoter region DNA (50 ng) was mixed with a 3 fold excess of competitor DNA (hlyR [33]) in a final volume of $20 \mu \mathrm{l}$, using the same binding buffer as described above. Increasing concentrations of $\mathrm{H}-\mathrm{NS}-\mathrm{His}$ were added as indicated, and the reaction was incubated at room temperature for $30 \mathrm{~min}$. The samples were resolved, stained and visualized as described above. Purified $\mathrm{H}$-NS-His protein was obtained as described [34], and the $h l y R$ DNA was obtained as described by [33].

\section{Results}

The invasion gene regulator coded by ygeH shares significant homology to EilA and HilA proteins

The complete genome sequence of E. coli 042 strain has led to the identification of two open reading frames encoding two proteins EilA (565 aminoacids) and YgeH (458 aminoacids) that exhibit similarity with the $S$. Typhimurium HilA protein (37\% and $29 \%$ of similarity with HilA, respectively). In addition to the already reported EilA regulator [19], the putative invasion gene regulator $\mathrm{YgeH}$ shows a significant degree of conservation (Figure 1). Whereas EilA appears to be restricted to E. coli 042 and other enteroaggregative strains, $\mathrm{YgeH}$ is also present in other $E$. coli strains, such as K12, enterohemorrhagic O157:H7, enteroaggregative hemorrhagic O104:H4 and others [18]. In spite of the significant degree of identity at the amino acid level between HilA and YgeH, no information is available about its biological activity, neither regulatory role nor its targets. Thus, we decided to further characterize this latter protein from strain 042.

\section{EilA and YgeH proteins from E. coli strain 042} complement the HilA ${ }^{-}$phenotype in S. Typhimurim strain SV5015

We addressed first the question whether the YgeH proteins from strain $042\left(\mathrm{YgeH}_{042}\right)$ and from strain $\mathrm{O} 157\left(\mathrm{YgeH}_{\mathrm{O} 157}\right)$ could be functionally equivalent to HilA. To test this, we first constructed a $\triangle$ hilA mutant of $S$. Typhimurium strain SV5015 (see Methods section). To monitor HilA activity we tested the expression of the sipA 


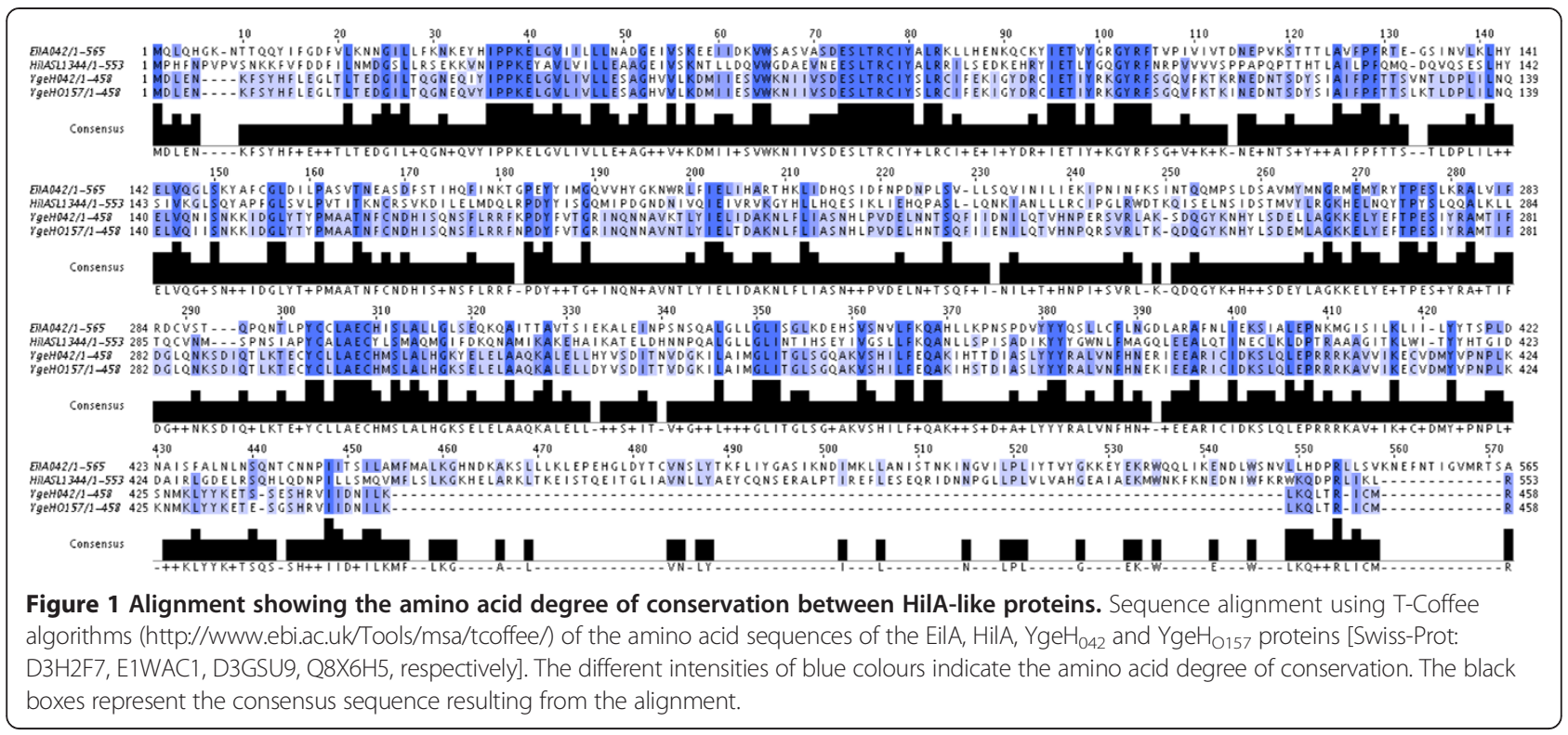

gene. The sipA gene is encoded in the sic/sip operon. The SipA effector is secreted by the type III secretion system encoded in the Salmonella pathogenicity island 1 (SPI1). Expression and translocation of SipA requires HilA activation of SPI1 operons [12,35]. To detect SipA, a 3xFLAG was fused to its C-terminal end. For the complementation studies, eilA and ygeH genes from strain 042 and the $y g e H$ gene from strain $\mathrm{O} 157$ were cloned in the expression vector pBAD18 [26]. Wild-type SV5015SipA (SipA::3xFLAG) and its $\Delta$ hilA derivative (SV5015 SipA::3xFLAG $\Delta$ hilA) were transformed with plasmids pBAD18, pBADHilA, pABDEilA, pBADYgeH ${ }_{042}$ and $\mathrm{pBADYgeH}_{\mathrm{O} 157}$. Cultures of the different transformants were grown in LB medium containing carbenicillin. Proteins present in the corresponding supernatants were analyzed by SDS-PAGE and Coomassie Brilliant Blue staining (Figure 2A). Moreover SipA::3xFLAG was immunodetected with anti-flag specific antibodies. As expected, SipA protein could only be detected in wt strain SV5015 SipA::3xFLAG, but not in the corresponding $\triangle$ hilA derivative (Figures $2 \mathrm{C}$ ). Alternatively, cultures of these strains were grown in LB medium containing carbenicillin and $0.2 \%$ arabinose. Overexpression of EilA, $\mathrm{YgeH}_{042}$, and $\mathrm{YgeH}_{\mathrm{O} 157}$ upon arabinose induction was confirmed by qRT-PCR. Upon arabinose inducing conditions, plasmids pBADHilA, pBADEilA, and

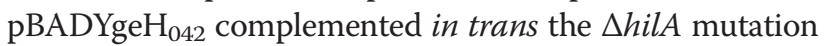
(Figure $2 \mathrm{~B}$ and $\mathrm{D}$ ). Interestingly, plasmid $\mathrm{pBADYgeH}_{\mathrm{O} 157}$ does not complement $\triangle$ hilA. The amount of expressed SipA::3xFLAG was very similar in strains harbouring either pBADHilA, pBADEilA or pBADYgeH. Other effector proteins could also be detected in cells expressing any of the three HilA-like modulators (Figure 2B). These results provide evidence for $E$. coli strain 042 EilA or $\mathrm{YgeH}$ proteins being able to functionally replace HilA in Salmonella, but not for $\mathrm{YgeH}_{\mathrm{O} 157}$. It is noteworthy that, compared to $\mathrm{YgeH}_{042}, \mathrm{YgeH}_{\mathrm{O} 157}$ presents 22 amino acid substitutions, that might result in loss of protein function (see Discussion section).

\section{EilA and $\mathrm{YgeH}_{042}$ activate sipA expression via InvF in Salmonella}

The regulatory cascade leading to $\operatorname{sip} A$ expression requires HilA-mediated activation of InvF which, in turn, activates SipA [36]. To check if either EilA or $\mathrm{YgeH}_{042}$ would influence $\operatorname{sip} A$ expression by activating InvF, strain SV5258 (invF::lacZ) was used. A $\triangle$ hilA derivative was constructed by transducing the $\triangle$ hilA:: $\mathrm{Cm}$ allele from strain MH-C1. Strain SV5258 was transformed with plasmid pBAD18, and strain SV5258hilA was transformed with plasmids pABD18, pBADHilA, pBADEilA and pBADYgeH ${ }_{042}$. The corresponding transformants were grown at $37^{\circ} \mathrm{C}$ to the beginning of the stationary phase $\left(\mathrm{OD}_{600} 2.0\right)$ either in $\mathrm{LB}$ medium or in LB medium containing $0.02 \%$ arabinose. As expected, neither arabinose nor the vector pBAD18 affected $i n v F$ expression in the hil $A^{+}$strain (Figure 3). In contrast, in $\triangle$ hilA mutant strain, the presence of plasmids pBADHilA, pBADEilA or pBADYgeH $\mathrm{H}_{042}$ drastically increased $i n v F$ transcription when cells were grown in LB medium containing arabinose (Figure 3). Hence, the effect of both EilA and $\mathrm{YgeH}_{042}$ influencing $i n v F$ transcription is similar to that of HilA.

\section{Regulation of $\mathrm{ygeH}_{042}$ expression}

We recently shed light on the role of the nucleoidassociated proteins $\mathrm{H}-\mathrm{NS}$ and Hha modulating hilA expression in Salmonella in a temperature-and growth phase-dependent manner. IHF appeared to antagonize 


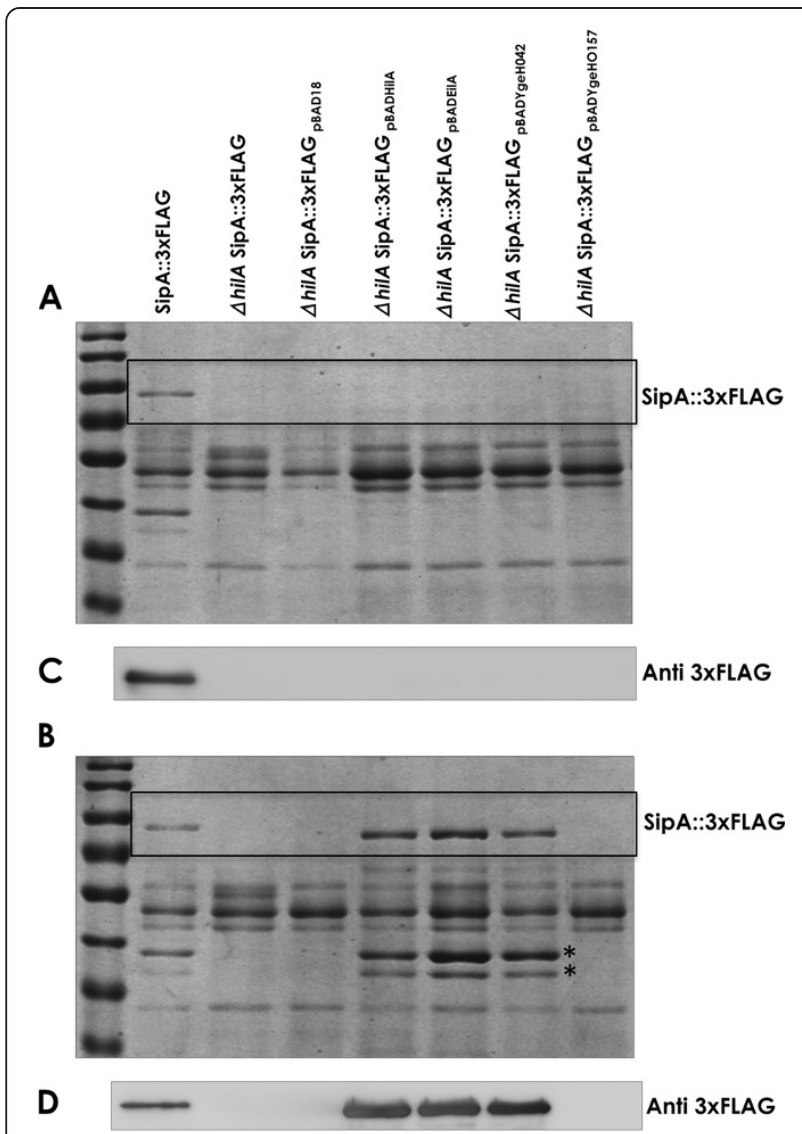

Figure 2 The HilA-like proteins EilA and YgeH from EAEC 042 are both able to complement in trans the $\triangle$ HilA phenotype in S. Typhimurium. Panel A and B: SDS-PAGE 10\% from whole secreted protein from strains MHS-2 (SV5015 sipA::3xFLAG) and MHS-3 (sipA::3xFLAG hilA) stained with Coomassie brilliant blue. C and $\mathbf{D}$ : Western blots from whole secreted protein from strains MHS-2 and MHS-3. A and C: Samples obtained from cultures grown in LB medium. B and $\mathbf{D}$ : Samples obtained from cultures grown in LB $0.2 \%$ L-arabinose. The SipA::3xFLAG protein was detected using monoclonal anti-flag antibody. The asterisks indicate the expression of other effector proteins according to [36], which are also activated by HilA-like proteins.

H-NS-mediated repression of hilA when cells reach the stationary phase of growth [20]. We decided to study the growth conditions influencing $y_{g e H_{042}}$ expression and the hypothetical role of the global modulator H-NS on $y \mathrm{geH}_{042}$ expression. We tried to construct a transcriptional ygeH::lacZ gene fusion in the chromosome of strain 042 . Under our working conditions, it was not possible to obtain this fusion in the 042 chromosome. We therefore decided to construct it in strain AAG1 (a $\Delta$ lacZ derivative from strain MG1655). Expression studies were performed in wt cells and in cells lacking the modulators $\mathrm{H}-\mathrm{NS}$ and/or IHF at varying temperatures and osmolarities, in exponentially growing cells as well as in cells entering the stationary phase. The most relevant regulatory

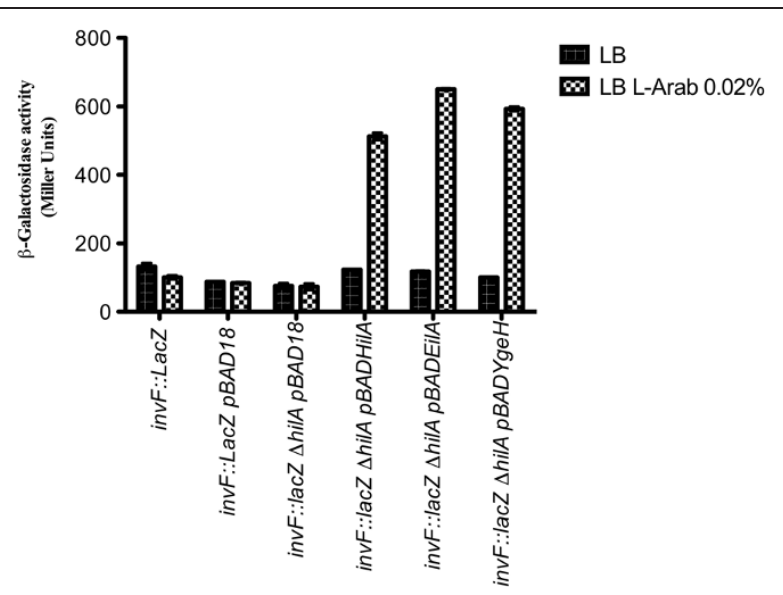

Figure 3 Expression of EilA and YgeH proteins induces invF expression. Transcription of invF in strains SV5258 and SV5258hilA was evaluated by measuring $\beta$-galactosidase activity in the different constructs. Samples were obtained from $L B C B_{100}$ or $L B C B_{100} L$-arabinose $0.02 \%$ cultures at the beginning of stationary phase (O.D.600 of 2.0) at $37^{\circ} \mathrm{C}$. Standard errors from three independent experiments are shown.

transcriptional data obtained in strain AAG1 were thereafter validated in strain 042 by detecting $\mathrm{YgeH}:: 3 x \mathrm{xLAG}$ by qRT-PCR and Western Blotting, as well (see below).

The expression profile of $y g \mathrm{eH}_{042}$ in different culture condition shows that stationary growth phase influences its expression, moderately increasing it. No effects of growth temperature or osmolarity are apparent (Figure 4A). With respect to the modulators H-NS and IHF, H-NS significantly represses $\mathrm{ygeH}_{042}$ expression in the different conditions tested, including the onset of the stationary phase. IHF appears to be required for a proper expression of $y g e H_{042}$. The fact that deletion of either ihfA or ihfB only decreases $y g^{2} \mathrm{H}_{042}$ expression when the $\mathrm{H}-\mathrm{NS}$ protein is available, suggest that, in the $y g^{2} H_{042}$ regulatory region, IHF might antagonize H-NS (Figure 4B and C).

To confirm that the observed regulatory effect of $\mathrm{H}-\mathrm{NS}$ on $y g e H_{042}$ expression in strain AGG1 also takes place in strain 042, yge $\mathrm{H}_{042}$ transcription was detected in strains 042 and $042 \Delta h n s$ by both real-time qRT-PCR and YgeH protein immunodetection. qRT-PCR shows an upregulation in the $\Delta h n s$ mutant of the $+4.5 \pm 0.17$ compared to wt strain. To detect $\mathrm{YgeH}_{042}, 3 x \mathrm{xLAG}$ was fused to its C-terminal end. The YgeH::3xFLAG was immunodectected by using the monoclonal anti-flag antibody. As expected from the results obtained in strain AAG1, expression of $y g e H_{042}$ increased in the absence of H-NS (Figure 5).

We studied next if $\mathrm{H}-\mathrm{NS}$ binds with specificity to $\mathrm{yeH}_{042}$ regulatory region. A virtual footprinting analysis of this region showed that it includes three putative sequences, predicted to be H-NS binding sites (see Additional file 2). A 228 bp DNA fragment that includes 




the $\mathrm{ygeH}_{042}$ promoter region was used for EMSA assays. According to the transcriptional data, binding of H-NS could be shown for the selected DNA fragment (Figure 6A). To provide evidence for specificity of binding, a competitive band shift experiment was performed, using both the fragment corresponding to the $\mathrm{ygeH}_{042}$ regulatory region, and a DNA fragment, corresponding to the regulatory region of the hly operon (407 bp). H-NS does not show specific binding to this fragment [33]. The competitive band shift assay showed that $\mathrm{H}-\mathrm{NS}$ preferentially binds to the $\mathrm{ygeH}_{042}$ regulatory region (Figure 6B).



Figure 5 The $\mathrm{H}-\mathrm{NS}$ protein represses the $y g e H$ gene at $37^{\circ} \mathrm{C}$ in strain 042. Immunodetection of $\mathrm{YgeH}:: 3 x \mathrm{FLAG}$ in strains $042 \mathrm{YgeH} 3 \mathrm{X}$ (YgeH::3xFLAG) and its isogenic mutant derivate 042YgeH3XHNS (YgeH::3XFLAG $\triangle h n s$ ). Cultures were grown in $L B$ medium at $37^{\circ} \mathrm{C}$ and samples were respectively collected at the logarithmic and onset of the stationary phase $\left(\mathrm{OD}_{600}\right.$ of 0.4 and 2.0$)$. Whole cell lysates $(5 \mu \mathrm{g})$ were resolved by $12.5 \%$ SDS-PAGE. The YgeH $:: 3 x$ FLAG protein was detected using the monoclonal anti-FLAG antibody. The GapdH protein was used as a protein loading control in western blot analysis and was detected using the monoclonal anti-GapdH antibody. The values shown under the strain name represent the relative amount of protein found in the mutant strains as compared to the wt strain (set as 1).

\section{YgeH upregulates ETT2 determinants}

Taking into account that YgeH is encoded in the ETT2 island, we decided to test the effect of YgeH depletion on expression of different ETT2 encoded genes. Genes selected corresponded to most of the ETT2-encoded operons, and were the $e i v F$ gene, encoding an InvF-like protein, eivA, etrA, eprH, ygeG, ygeK and yqeI (locus tag: EC042_3070, EC042_3060, EC042_3059, EC042_3049, EC042_3053, EC042_3045, respectively). Expression of these genes was first monitored by qRT-PCR in the wt 042 strain and in the geH $_{042}$ single mutant. As expected from YgeH being a transcriptional activator, depletion of ygeH resulted in downregulation of the different ETT2 genes (Figure 7A). Taking into account that ygeH expression is increased in an hns mutant, we decided to further investigate whether, as a response to reduced H-NS levels, ETT2 genes are also upregulated in a $\Delta h n s$ mutant. As expected, all the ETT2 genes tested are upregulated in strain $042 \Delta h n s$ (Figure 7B). Hence, expression of ETT2 genetic determinants is subjected to $\mathrm{H}$-NS-mediated repression via YgeH.

\section{Discussion}

In this report we provide evidence for the close relationship between the E. coli $042 \mathrm{YgeH}$ protein, the EilA regulator and the Salmonella regulator HilA. Genomic studies have shown that the ETT2 island is widespread in Escherichia and Shigella strains $[18,37,38]$, although in many of them the gene cluster is either disrupted or incomplete [18]. The YgeH protein, which shares significant similarity to the SPI1 modulator HilA, is present in several of the strains analyzed as, for instance, 042, O157:H7 or MG1655. We show here that the $\mathrm{YgeH}$ protein is functional in strain 042. Furthermore, the YgeH protein, as well as the already 


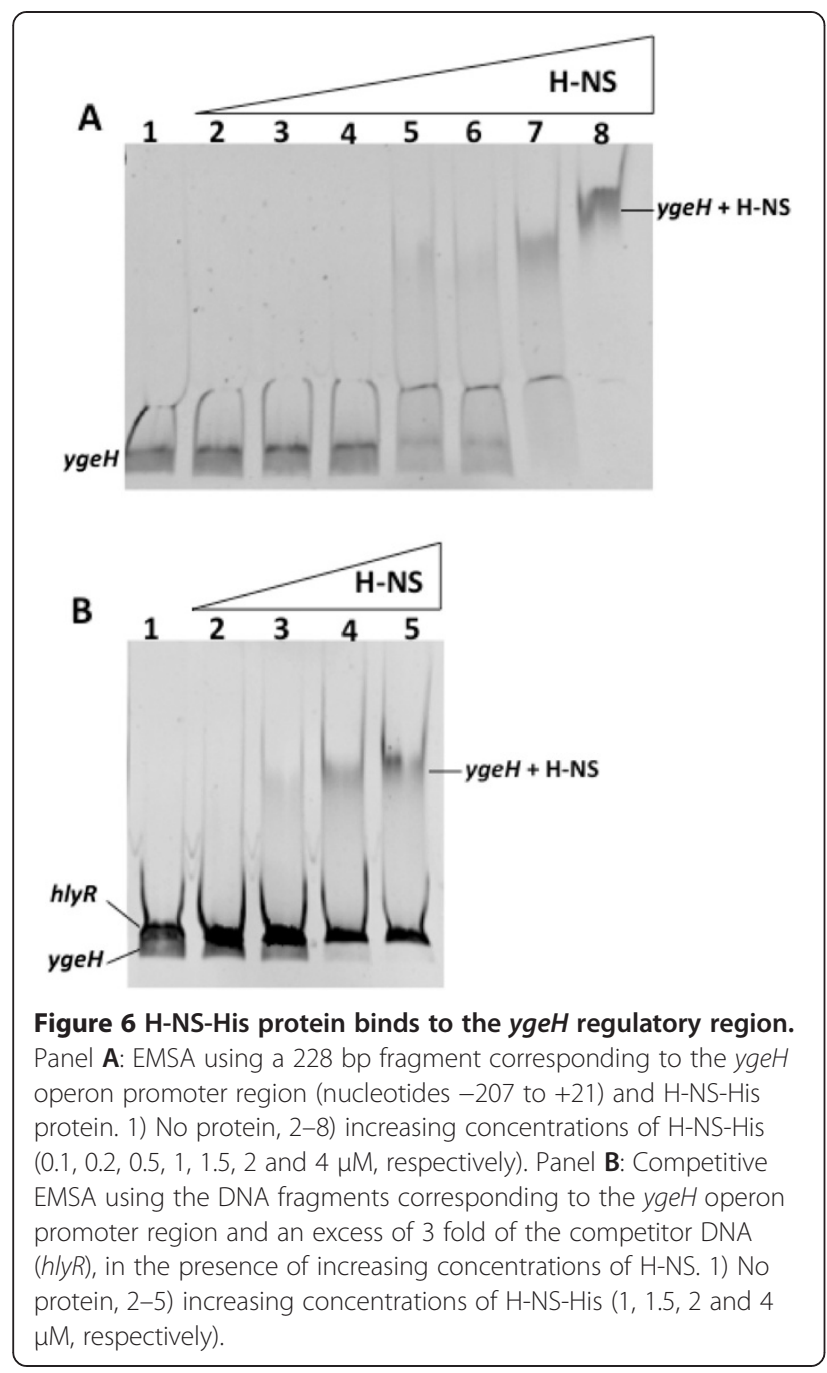

characterized EilA protein, is able to complement the $\Delta$ hilA mutation in Salmonella. All of them target the SPI1 gene $i n v F$ which, in turn, activates the expression of effectors such as SipA. These results support the close functional and structural relationship between these proteins and suggest a common origin. Remarkably, whereas either $\mathrm{YgeH}_{042}$ or EilA complement the hilA mutation, $\mathrm{YgeH}_{\mathrm{O} 157}$ does not. The amino acid sequence of $\mathrm{YgeH}_{042}$ and of $\mathrm{YgeH}_{\mathrm{O} 157}$ differs in several amino acid residues, and these differences may be underlying loss of protein function.

Unlike eilA gene [19], ygeH is expressed when 042 cells grow in LB medium. Unlike hilA, significant expression levels are detected both at the exponential and stationary growth phases. Only a moderate increase in transcription is apparent at the onset of the stationary phase. When growing in LB medium, neither temperature nor osmolarity are critical factors altering yge $\mathrm{H}_{042}$ expression. Remarkably, $\mathrm{H}-\mathrm{NS}$ appears as a repressor of $y g e \mathrm{H}_{042}$ expression. Under

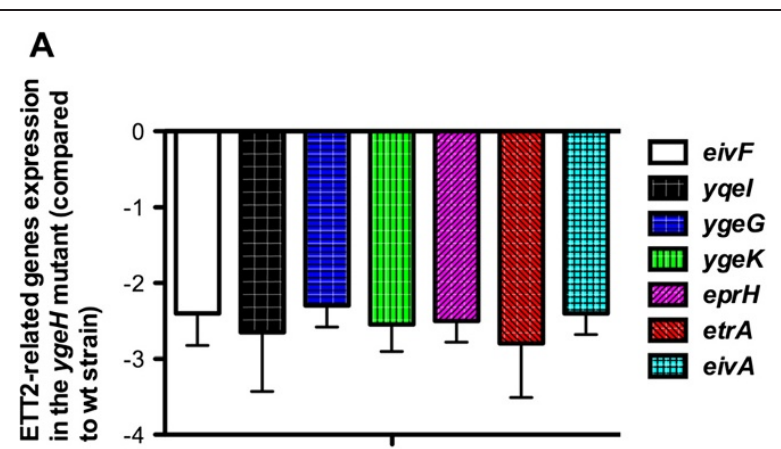

B

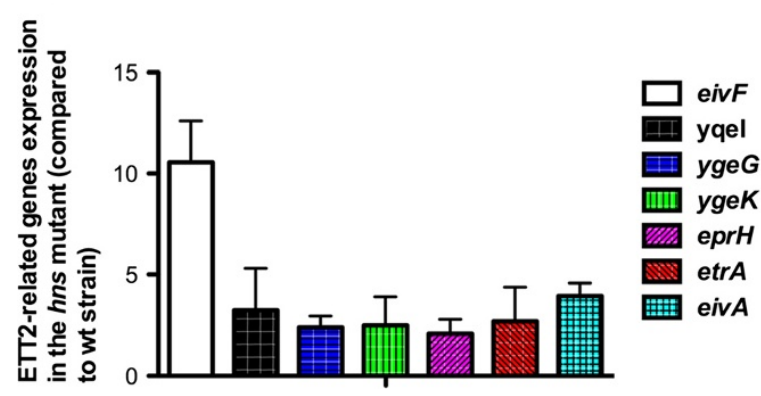

Figure 7 Effect of $\triangle y g e H$ and $\Delta h n s$ mutations on the expression of ETT2 genes from EAEC 042. Expression of ETT2-related genes was determined by reverse transcription and GRT-PCR. Data show the transcription levels of ETT2-related genes in bacteria cultured in LB broth to early-stationary phase (O.D.600 of 2.0). As internal standard the gapA gene was used. Data are means of three independent experiments. Standard deviations are shown. Panel A: Relative expression of the $\Delta y g e H$ mutant relative to wt strain. Panel B: Relative expression of the $\Delta h n s$ mutant compared to wt strain.

all growth conditions tested, the hns mutant from strain 042 showed an upregulated expression of $y g e H$. Whereas these data show that $\mathrm{H}-\mathrm{NS}$ is a repressor of $\mathrm{ygeH}_{042}$, it is also apparent that environmental conditions leading to a full induction of $y g^{2} H_{042}$ (i.e., environmental conditions which would result in similar expression levels of $y g e H_{042}$ in both wt 042 strain and the hns mutant) are not among those used in this work. H-NS targeting yge $\mathrm{H}_{042}$ is also supported by the EMSA assays in the presence of a DNA fragment including the $y g e H$ regulatory region and a competitor DNA molecule. Altogether, these data show that, when coping with the appropriate environmental conditions, 042 cells must be able to significantly increase YgeH levels by counteracting H-NS silencing.

A question to be addressed is the biological role of the either complete or incomplete ETT2 determinants in different E. coli isolates, ranging from MG1655 to O157: H7. Some previous data together with the results presented in this paper may help to clarify this. In a previous report [39], ygeH, etrA and eivF genes from the ETT2 genetic element from E. coli $\mathrm{O} 157: \mathrm{H} 7$ were mutagenized, and the effect of the corresponding mutant alleles on expression of 
the LEE (locus of enterocyte effacement) pathogenicity island was studied. Whereas disruption of the $\operatorname{ygeH}_{\mathrm{O} 157}$ gene did not influence LEE expression (and hence it was not further characterized), the etrA and eivF mutant alleles were found to significantly enhance secretion of proteins encoded by the LEE. Transcription of several LEE genes was altered in these mutants. We show in this report that, unlike $\mathrm{YgeH}_{042}, \mathrm{YgeH}_{\mathrm{O} 157}$ fails to complement the $\Delta$ hilA mutation. The production of a non-functional YgeH protein in E. coli strain $\mathrm{O} 157$ may be underlying the fact that, whereas etrA and eivF mutants of strain O157 influence LEE expression, ygeH mutants do not [39]. Hence, mutational attrition that occurs in some genomic islands may also affect some of the encoded modulators, thus resulting in a complex array of functional and non-functional genes that may significantly increase the genomic plasticity of those strains that incorporate the corresponding genomic islands.

\section{Conclusions}

YgeH and EilA proteins can functionally replace HilA in Salmonella. E. coli $042 \mathrm{YgeH}$ protein expression is similar when cells grow in a wide variety of lab culture conditions. Whereas YgeH expression upregulates expression of ETT2 genes, H-NS downregulates their expression. As exemplified by $\mathrm{YgeH}_{\mathrm{O} 157}$ protein, some of the YgeH modulators encoded in ETT2 islands may be non-functional. Hence, mutational attrition identified in the ETT2 cluster is not restricted to YgeH-modulated genes, but may also affect the modulator itself.

\section{Additional files}

Additional file 1: Sequence of the oligonucleotides utilized in this study.

Additional file 2: Putative H-NS binding sites found in the regulatory region of the $\mathrm{ygeH}_{042}$ gene.

\section{Abbreviations \\ SPI1: Salmonella pathogenicity island 1; TTSS: Type 3 secretion system; ETT2: Escherichia coli type 3 secretion system 2; LEE: Locus of enterocyte effacement; Km: Kanamycin; CB: Carbenicillin; Cm: Choramphenicol; Sm: Streptomycin; Tc: Tetracycline; TCA: Trichloroacetic acid.}

\section{Competing interests}

The authors declare that they have no competing interests.

\section{Authors' contributions}

Conceived and designed the experiments: $\mathrm{MH}$ and AJ. Performed the experiments: MH, MD, SP. Analysed the data: MH, MD, SP and AJ. Wrote the manuscript: $\mathrm{MH}$ and AJ. All authors read and approved the final manuscript.

\section{Acknowledgements}

This work was supported by funds from the Spanish MICINN-FEDER (BFU2010-21836-C02-01 and CSD2008-00013) and the Generalitat de Catalunya (2009SGR66). We thank Prof. Josep Casadesús (University of Seville) and Prof. lan Henderson (University of Birmingham) for providing strains.
Received: 2 June 2014 Accepted: 14 October 2014



\section{References}

1. Wardlaw T, Salama P, Brocklehurst C, Chopra M, Mason E: Diarrhoea: why children are still dying and what can be done. Lancet 2010, 375:870-872.

2. Ruchaud-Sparagano M-H, Maresca M, Kenny B: Enteropathogenic Escherichia coli (EPEC) inactivate innate immune responses prior to compromising epithelial barrier function. Cell Microbiol 2007, 9:1909-1921.

3. Kaper JB, Nataro JP, Mobley HL: Pathogenic Escherichia coli. Nat Rev Microbiol 2004, 2:123-140.

4. Nataro JP, Kaper JB, Robins-Browne R, Prado V, Vial P, Levine MM: Patterns of adherence of diarrheagenic Escherichia coli to HEp-2 cells. Pediatr Infect Dis J 1987, 6:829-831.

5. Harrington SM, Dudley EG, Nataro JP: Pathogenesis of enteroaggregative Escherichia coli infection. FEMS Microbiol Lett 2006, 254:12-18.

6. Nataro JP, Deng Y, Cookson S, Cravioto A, Savarino SJ, Guers LD, Levine MM, Tacket CO: Heterogeneity of enteroaggregative Escherichia coli virulence demonstrated in volunteers. J Infect Dis 1995, 171:465-468.

7. Huang DB, Nataro JP, DuPont HL, Kamat PP, Mhatre AD, Okhuysen PC, Chiang T: Enteroaggregative Escherichia coli is a cause of acute diarrheal illness: a meta-analysis. Clin Infect Dis 2006, 43:556-563.

8. Estrada-Garcia T, Navarro-Garcia F: Enteroaggregative Escherichia coli pathotype: a genetically heterogeneous emerging foodborne enteropathogen. FEMS Immunol Med Microbiol 2012, 66:281-298.

9. Okeke IN, Wallace-Gadsden F, Simons HR, Matthews N, Labar AS, Hwang J, Wain J: Multi-locus sequence typing of enteroaggregative Escherichia coli isolates from Nigerian children uncovers multiple lineages. PLOS One 2010, 5:e14093.

10. Chaudhuri RR, Sebaihia M, Hobman JL, Webber MA, Leyton DL, Goldberg MD, Cunningham AF, Scott-Tucker A, Ferguson PR, Thomas CM, Frankel G, Tang CM, Dudley EG, Roberts IS, Rasko DA, Pallen MJ, Parkhill J, Nataro JP, Thomson NR, Henderson IR: Complete genome sequence and comparative metabolic profiling of the prototypical enteroaggregative Escherichia coli strain 042. PloS One 2010, 5:e8801.

11. Altier C: Genetic and environmental control of salmonella invasion. J Microbiol 2005, 43:85-92

12. Ellermeier JR, Slauch JM: Adaptation to the host environment: regulation of the SPI1 type III secretion system in Salmonella enterica serovar Typhimurium. Curr Opin Microbiol 2007, 10:24-29.

13. Lee $C A$, Jones $B D$, Falkow $S$ : Identification of a Salmonella typhimurium invasion locus by selection for hyperinvasive mutants. Proc Natl Acad SCi U S A 1992, 89:1847-1851.

14. Bajaj V, Hwang C, Lee CA: hilA is a novel ompR/toxR family member that activates the expression of Salmonella typhimurium invasion genes. Mol Microbiol 1995, 18:715-727.

15. Jones BD, Falkow S: Identification and characterization of a Salmonella typhimurium oxygen-regulated gene required for bacterial internalization. Infect Immun 1994, 62:3745-3752

16. Bajaj V, Lucas RL, Hwang C, Lee CA: Co-ordinate regulation of Salmonella typhimurium invasion genes by environmental and regulatory factors is mediated by control of hilA expression. Mol Microbiol 1996, 22:703-714.

17. Chubiz JEC, Golubeva YA, Lin D, Miller LD, Slauch JM: FliZ regulates expression of the Salmonella pathogenicity island 1 invasion locus by controlling HilD protein activity in Salmonella enterica serovar typhimurium. J Bacteriol 2010, 192:6261-6270.

18. Ren C-P, Chaudhuri RR, Fivian A, Bailey CM, Antonio M, Barnes WM, Pallen MJ: The ETT2 gene cluster, encoding a second type III secretion system from Escherichia coli, is present in the majority of strains but has undergone widespread mutational attrition. J Bacteriol 2004, 186:3547-3560.

19. Sheikh J, Dudley EG, Sui B, Tamboura B, Suleman A, Nataro JP: EilA, a HilA-like regulator in enteroaggregative Escherichia coli. Mol Microbio/ 2006, 61:338-350.

20. Queiroz MH, Madrid C, Paytubi S, Balsalobre C, Juárez A: Integration host factor alleviates H-NS silencing of the Salmonella enterica serovar Typhimurium master regulator of SPI1, hilA. Microbiol (Reading, Engl) 2011, 157:2504-2514.

21. Bullas LR, Ryu J: Salmonella typhimurium LT2 strains which are $r-m+$ for all three chromosomally located systems of DNA restriction and modification. J Bacteriol 1983, 156:471-474. 
22. Aberg A, Shingler $V$, Balsalobre $C$ : Regulation of the fimB promoter: a case of differential regulation by ppGpp and DksA in vivo. Mol Microbiol 2008, 67:1223-1241

23. Datsenko KA, Wanner BL: One-step inactivation of chromosomal genes in Escherichia coli K-12 using PCR products. Proc Natl Acad Sci U S A 2000, 97:6640-6645.

24. Cherepanov PP, Wackernagel W: Gene disruption in Escherichia coli: TcR and $\mathrm{KmR}$ cassettes with the option of Flp-catalyzed excision of the antibiotic-resistance determinant. Gene 1995, 158:9-14.

25. Uzzau S, Figueroa-Bossi N, Rubino S, Bossi L: Epitope tagging of chromosomal genes in Salmonella. Proc Natl Acad Sci U S A 2001, 98:15264-15269.

26. Guzman LM, Belin D, Carson MJ, Beckwith J: Tight regulation, modulation, and high-level expression by vectors containing the arabinose PBAD promoter. J Bacteriol 1995, 177:4121-4130.

27. Simons RW, Houman F, Kleckner N: Improved single and multicopy lac-based cloning vectors for protein and operon fusions. Gene 1987, 53:85-96.

28. Sambrook J: Molecular Cloning: a Laboratory Manual, (3 Volume Set). 3rd edition. Cold Spring Harbour, NY: Cold Spring Harbor Laboratory Press; 2001.

29. Schmieger H: Phage P22-mutants with increased or decreased transduction abilities. Mol Gen Genet 1972, 119:75-88.

30. Powell BS, Rivas MP, Court DL, Nakamura Y, Turnbough CL: Rapid confirmation of single copy lambda prophage integration by PCR. Nucleic Acids Res 1994, 22:5765-5766.

31. Miller JH: A Short Course in Bacterial Genetics: a Laboratory Manual and Handbook for Escherichia Coli and Related Bacteria. Cold Spring Harbour NY: Cold Spring Harbour Laboratory Press; 1992.

32. Dietrich M, Mollenkopf $H$, So M, Friedrich A: Pilin regulation in the pilT mutant of Neisseria gonorrhoeae strain MS11. FEMS Microbiol Lett 2009, 296:248-256.

33. Madrid C, Nieto JM, Paytubi S, Falconi M, Gualerzi CO, Juárez A: Temperature- and H-NS-dependent regulation of a plasmid-encoded virulence operon expressing Escherichia coli hemolysin. J Bacteriol 2002, 184:5058-5066.

34. Nieto JM, Carmona M, Bolland S, Jubete Y, de La Cruz F, Juárez A: The hha gene modulates haemolysin expression in Escherichia coli. Mol Microbiol 1991, 5:1285-1293

35. Lostroh CP, Bajaj V, Lee CA: The cis requirements for transcriptional activation by HilA, a virulence determinant encoded on SPI-1. Mol Microbiol 2000, 37:300-315.

36. Darwin $\mathrm{KH}$, Miller VL: InvF is required for expression of genes encoding proteins secreted by the SPI1 type III secretion apparatus in Salmonella typhimurium. J Bacteriol 1999, 181:4949-4954.

37. Makino S-I, Tobe T, Asakura H, Watarai M, Ikeda T, Takeshi K, Sasakawa C: Distribution of the secondary type III secretion system locus found in enterohemorrhagic Escherichia coli 0157:H7 isolates among Shiga toxin-producing E. coli strains. J Clin Microbiol 2003, 41:2341-2347.

38. Osawa K, Shibata M, Nishiyama Y, Kurokawa M, Yamamoto G, Kinoshita S, Kataoka N: Identification of the ETT2 locus in human diarrheagenic Escherichia coli by multiplex PCR. J Infect Chemother 2006, 12:157-159.

39. Zhang L, Chaudhuri RR, Constantinidou C, Hobman JL, Patel MD, Jones AC, Sarti D, Roe AJ, Vlisidou I, Shaw RK, Falciani F, Stevens MP, Gally DL, Knutton S, Frankel G, Penn CW, Pallen MJ: Regulators encoded in the Escherichia coli type III secretion system 2 gene cluster influence expression of genes within the locus for enterocyte effacement in enterohemorrhagic E. coli 0157:H7. Infect Immun 2004, 72:7282-7293.

\section{doi:10.1186/s12866-014-0268-5}

Cite this article as: Hüttener et al:: HilA-like regulators in Escherichia coli pathotypes: the YgeH protein from the enteroaggregative strain 042. BMC Microbiology 2014 14:268.

\section{Submit your next manuscript to BioMed Central and take full advantage of:}

- Convenient online submission

- Thorough peer review

- No space constraints or color figure charges

- Immediate publication on acceptance

- Inclusion in PubMed, CAS, Scopus and Google Scholar

- Research which is freely available for redistribution

Submit your manuscript at www.biomedcentral.com/submit 